Homology, Homotopy and Applications, vol.13(2), 2011, pp.43-62

\title{
RATIONAL HOMOTOPY MODELS FOR TWO-POINT CONFIGURATION SPACES OF LENS SPACES
}

\author{
MATTHEW S. MILLER \\ (communicated by Gunnar Carlsson)
}

\begin{abstract}
We study the algebraic topology of configuration spaces as interesting objects in their own right and with the goal of constructing invariants for topological manifolds. We calculate the complete Massey product structure for the universal cover of the space of two point configurations in a three-dimensional lens space. We then construct rational homotopy models for these spaces and calculate the rational homotopy groups.
\end{abstract}

\section{Introduction}

\subsection{Configuration Spaces}

The objects we study are configuration spaces, which parametrize collections of $k$ distinct labelled points free to move through a given space.

Definition 1.1. The configuration space of $k$ points in a manifold $M$ is the space

$$
\operatorname{Conf}_{k}(M):=\left\{\left(x_{1}, x_{2}, \ldots, x_{k}\right) \in M^{\times k}: x_{i} \neq x_{j} \text { for } i \neq j\right\} .
$$

Configuration spaces have played an important role in many areas of topology, ranging from operads to iterated loop spaces to knot theory. They have also been studied for their own sake, and in general it is not known how to compute the cohomology ring of configurations in an arbitrary manifold, see Bendersky and Gitler [3], Cohen and Taylor [4], Felix and Thomas [5], and Totaro [11].

A long standing conjecture about configuration spaces was that two homotopy equivalent manifolds of the same dimension would have homotopy equivalent configuration spaces. Lambrechts and Stanley [7] resolved this conjecture for the rational homotopy type of a configuration of two points in a closed two-connected manifold. In [8], Longoni and Salvatore used Lens spaces to provide the first counterexample. One important motivation for studying configuration spaces is thus that the homotopy type of a configuration space can be sensitive to the more subtle homeomorphism type of the original manifold. As in Felix and Thomas [5] and Longoni and Salvatore $[\mathbf{8}]$ we find that Massey products play a central role in our study of configuration spaces.

Received September 11, 2008, revised November 4, 2010; published on September 21, 2011. 2000 Mathematics Subject Classification: 57N65, 55P62, 55S30, 57N75.

Key words and phrases: homology, homotopy, Massey product, lens space, configuration space. Article available at http://intlpress.com/HHA/v13/n2/a4 and doi:10.4310/HHA.2011.v13.n2.a4 Copyright (C) 2011, International Press. Permission to copy for private use granted. 


\subsection{Lens Spaces}

The first class of manifolds used to illustrate the distinction between homeomorphism and homotopy equivalence was Lens spaces, see Reidemeister [10]. To define Lens spaces, let the group $\mathbb{Z}_{p}$ be the group of $p^{t h}$ roots of unity in $\mathbb{C}$, generated by $\zeta=e^{2 \pi i / p}$. Note that $p$ need not be prime. Let $S^{3}$ be the submanifold of unit length vectors in $\mathbb{C}^{2}$. Define the action of $\zeta_{q}$ by

$$
\zeta_{q}\left(z_{1}, z_{2}\right)=\left(\zeta z_{1}, \zeta^{q} z_{2}\right) .
$$

Definition 1.2. For the relatively prime pair of integers, $(p, q)$ we define the Lens space $L(p, q)$ to be the quotient $S^{3} /\left\langle\zeta_{q}\right\rangle$.

Theorem 1.3. (Reidemeister [10]) The Lens spaces $L(p, q)$ and $L\left(p, q^{\prime}\right)$ are homotopy equivalent if and only if for some $r \in \mathbb{Z}_{p}$

$$
q^{\prime} \equiv \pm q r^{2} \quad \bmod p .
$$

The Lens spaces $L(p, q)$ and $L\left(p, q^{\prime}\right)$ are homeomorphic if and only if

$$
q^{\prime} \equiv \pm q^{ \pm 1} \quad \bmod p
$$

\subsection{Overview}

As stated before, the main goal of our work is to model the rational homotopy type of certain configuration spaces. We extend the approach of Longoni and Salvatore $[8]$ to study the universal cover of the configuration space $\operatorname{Conf}_{2}(L(p, q))$, which we denote by $\widetilde{\operatorname{Conf}}{ }_{2}(L(p, q))$. Our first main theorem is Theorem 3.2 which is a computation of all of the Massey products for $\widetilde{\operatorname{Conf}}{ }_{2}(L(p, q))$. We use the Massey product structure to prove our second main result, Theorem 4.2 , which is the construction of a differential graded algebra, $A(p, q)$, that is a model for the co-chains on $\widetilde{\operatorname{Conf}}_{2}(L(p, q))$.

Longoni and Salvatore $[8]$ show that $\widetilde{\operatorname{Conf}} 2(L(7,1))$ is homotopy equivalent to $\left(\vee_{6} S^{2}\right) \times S^{3}$ and hence is rationally formal and has no non-trivial Massey products. Then they proceed to construct representing submanifolds for the cohomology classes of $\widetilde{\operatorname{Conf}}_{2}(L(7,2))$ and use these representatives to show that there is a non-trivial Massey product in the cohomology of $\widetilde{\operatorname{Conf}}{ }_{2}(L(7,2))$. At the end of their paper they suggest that it would be interesting to study the homeomorphism type of all Lens spaces through the rational homotopy type of configuration spaces. While initially we use the constructions and techniques of Longoni and Salvatore [8], which in some cases have direct generalizations as mentioned at the end of [8], at our level of generality there are new technical difficulties. In particular, there are several transversality problems to overcome. Moreover, Longoni and Salvatore [8] compare only two Massey product structures, one of which is entirely trivial, leaving the task of showing that in the other there is a non-trivial product. When attempting to understand the class of all spaces, $\widetilde{\operatorname{Conf}_{2}}(L(p, q))$, many of which admit non-trivial products, the algebraic problem of finding invariants of the Massey product structure remains open and is a topic of our current work. During the summer of 2010 at an intensive research period on configuration spaces at the Centro di Ricerca Matematica Ennio De Giorgio, we were happy to hear a lecture by Paolo Salvatore. In part of his lecture he 
used the work contained in this paper to distinguish the rational homotopy type of $\widetilde{\operatorname{Conf}}_{2}(L(11,2))$ and $\widetilde{\operatorname{Conf}}_{2}(L(11,3))$.

An interesting question raised by the anonymous referee is that of the integral homotopy type of $\widetilde{\operatorname{Conf}}_{2}(L(p, q))$. We are currently investigating whether the rational models constructed here are integral homotopy models for $\widetilde{\operatorname{Conf}_{2}}(L(p, q))$. Anick's work in $[\mathbf{1}, \mathbf{2}]$ provides one method by which to approach this problem. It may also be interesting to attempt a computation of the Steenrod operations directly.

Our plan is as follows. In Section 2 we discuss the cohomology of $\widetilde{\operatorname{Conf}} 2(L(p, q))$ and how to represent cohomology classes geometrically. In Section 3 we compute all Massey products in $\widetilde{\operatorname{Conf}} 2(L(p, q))$. To do this we make use of intersection theory and describe explicit perturbations of the cohomology representatives in order to make intersections transversal. Section 4 focuses on the algebraic theorems. We use the Massey product information from Section 3 to construct the algebras $A(p, q)$, which are rational models for the co-chains on $\widetilde{\operatorname{Conf}}_{2}(L(p, q))$.

\section{The cohomology ring and geometric representatives}

In this section we discuss the cohomology of the universal cover of two point configuration spaces of Lens spaces. In particular, we consider

$$
\widetilde{\operatorname{Conf}}_{2}(L(p, q))=\left\{(x, y) \in S^{3} \times S^{3}: x \neq \zeta_{q}^{k} y \text { for all } k \in \mathbb{Z}_{p}\right\} .
$$

This space can be written as $\left(S^{3} \times S^{3}\right) \backslash \Delta_{q}$, where $\Delta_{q}$ is a union of diagonals $\Delta_{q}=$ $\cup_{k \in \mathbb{Z}_{p}} \Delta_{q}^{k}$ and $\Delta_{q}^{k}$ is the image of the embedding

$$
\begin{aligned}
S^{3} \longrightarrow S^{3} \times S^{3} \\
w \longmapsto\left(w, \zeta_{q}^{k} w\right) .
\end{aligned}
$$

We also describe two standard models for $S^{3}$ which are useful in understanding the cohomology geometrically.

Recall from the calculation of Longoni and Salvatore in [8], that

$$
H^{*}\left(\widetilde{\operatorname{Conf}_{2}}(L(p, q))\right)= \begin{cases}\mathbb{Z}^{p-1} & *=2 \\ \mathbb{Z} & *=3 \\ \mathbb{Z}^{p-1} & *=5,\end{cases}
$$

which follows from an easy Leray-Serre spectral sequence calculation that also determines the ring structure. Lefshetz Duality gives the following isomorphism

$$
H_{6-*}\left(S^{3} \times S^{3}, \Delta_{q}\right)=H^{*}\left(\widetilde{\operatorname{Conf}}_{2}(L(p, q))\right) .
$$

For convenience we list the generators of $H_{*}\left(S^{3} \times S^{3}, \Delta_{q}\right)$ and their Lefshetz duals in Table 1 . The subscripts $k$ are in $\mathbb{Z}_{p}$. With this notation we describe the cohomology ring as the differential graded algebra generated in degree two by the $a_{k}$ and in degree three by $\eta$ with the relations $\left[a_{j}\right] \cdot\left[a_{k}\right]=0$ and $\sum_{k \in \mathbb{Z}_{p}}\left[a_{k}\right]=0$. This description of the cohomology ring is symmetric with respect to the action of $\mathbb{Z}_{p}$ on subscripts.

To describe submanifolds that represent generators of $H_{*}\left(S^{3} \times S^{3}, \Delta_{q}\right)$, we use the standard descriptions of $S^{3}$, given through its standard embedding as the unit sphere in $\mathbb{C}^{2}$ and through its canonical Heegard decomposition. 


\begin{tabular}{|c|c|c|c|}
\hline * & $H^{*}$ & $\begin{array}{ll}1 & H_{6-*} \\
\text { I }\end{array}$ & $6-*$ \\
\hline 2 & {$\left[a_{k}\right]$} & $\left.\underset{\mathrm{I}}{\mathrm{I}} \longrightarrow A_{k}\right]$ & 4 \\
\hline 3 & {$[\eta]<$} & $\longrightarrow[S]$ & 3 \\
\hline 5 & {$\left[\eta a_{k}\right]$} & $\stackrel{\mathrm{I}}{\longrightarrow}\left[S \cap A_{k}\right]$ & 1 \\
\hline
\end{tabular}

Table 1: The generators of $H_{*}\left(S^{3} \times S^{3}, \Delta_{q}\right)$ and their Lefshetz duals.

\subsection{The Complex Model}

Using the standard embedding of $S^{3}$ in $\mathbb{C}^{2}$ we have

$$
\begin{aligned}
\widetilde{\operatorname{Conf}}_{2}(L(p, q)) & =S^{3} \times S^{3} \backslash \Delta_{q} \\
& =\left\{\left(z_{1}, z_{2}\right) ;\left(z_{3}, z_{4}\right) \in \mathbb{C}^{2} \times \mathbb{C}^{2}:\left(z_{1}, z_{2}\right) \neq\left(\zeta^{k} z_{3}, \zeta^{q k} z_{4}\right)\right\} .
\end{aligned}
$$

The representatives for the classes $\left[A_{k}\right]$ will be denoted $A_{k}$ and can be thought of as graphs of "fattened" functions from $S^{3}$ to itself. That is, for every point in the first $S^{3}$ factor, $A_{k}$ contains an interval in the second $S^{3}$ which goes between the diagonals $\Delta_{q}^{k-1}$ and $\Delta_{q}^{k}$. Since we often make use of objects of this form, we make the following definition.

Definition 2.1. Given a map $f: X \times Y \rightarrow X$ we define the track of $f$, denoted $\Gamma(f)$, to be the image of $\left(i d_{X} \circ \pi\right) \times f: X \times Y \rightarrow X \times X$, where $i d_{X}$ is the identity map on $X$ and $\pi$ the canonical projection from $X \times Y$ to $X$.

Note that the graph of a self map is the special case of a track in which $Y$ is a point.

We also set $I(k)$ to be the interval $(k-1, k)$, and note that $I(1)=I=(0,1)$. We choose the canonical positive orientation on $I(k)$. Throughout we choose the orientation for $S^{3}$ that is consistent with the orientation at the point $(1,0)$ given by the basis tangent vectors $(i, 0),(1,0)$ and $(0, i)$.

Using our notion of a track we define $A_{k}$ to be the same submanifold as that given by Longoni and Salvatore in $[\mathbf{8}]$.

Definition 2.2. Let $A_{k}$ be the track $\Gamma\left(\alpha_{k}\right)$, where

$$
\begin{aligned}
\alpha_{k}: S^{3} \times I(k) & \longrightarrow S^{3} \\
(w, s) & \longmapsto \zeta_{q}{ }^{s} w .
\end{aligned}
$$

Alternatively, in coordinates, $w=\left(z_{1}, z_{2}\right)$ and

$$
A_{k}=\left\{\left(z_{1}, z_{2}\right) ;\left(\zeta^{s} z_{1}, \zeta^{q s} z_{2}\right): s \in I(k)\right\} \subset S^{3} \times S^{3} \subset \mathbb{C}^{2} \times \mathbb{C}^{2} .
$$

To show that these submanifolds do represent homology generators and the sum of these classes is zero, we consider a portion of the long exact sequence of the pair 
$\left(S^{3} \times S^{3}, \Delta_{q}\right)$. Let $\bar{A}_{k}$ be the closure of $A_{k}$ in $S^{3} \times S^{3}$ and consider the following.

$$
\begin{array}{r}
H_{4}\left(S^{3} \times S^{3}\right)=0 \longrightarrow H_{4}\left(S^{3} \times S^{3}, \Delta_{q}\right) \stackrel{\partial}{\longrightarrow} H_{3}\left(\Delta_{q}\right) \cdots \\
{\left[\bar{A}_{k}\right] \longmapsto\left[\Delta_{q}^{k}\right]-\left[\Delta_{q}^{k-1}\right] .}
\end{array}
$$

From the injectivity of $\partial$ it is immediate that the $\left[\bar{A}_{k}\right]$ generate $H_{4}\left(S^{3} \times S^{3}, \Delta_{q}\right)$ and that $\sum_{k \in \mathbb{Z}_{p}}\left[\bar{A}_{k}\right]=0$. Throughout the rest of this paper we use the locally finite representatives $A_{k}$ so as to avoid issues of transversality on the boundary.

The generator of $H_{3}\left(S^{3} \times S^{3}, \Delta_{q}\right)$ is represented by an embedded $S^{3}$.

Definition 2.3. Define $S$ to be the image of the embedding $S^{3} \rightarrow S^{3} \times S^{3}$ which sends $w$ to $(1, w)$.

The point in the first coordinate may be chosen arbitrarily, but our choice of $(1,0)$ makes some computations easier.

By Lefshetz duality the generators of $H_{1}\left(S^{3} \times S^{3}, \Delta_{q}\right)$ are given by homology classes of the intersections,

$$
S \cap A_{k}=\left\{\alpha_{k}((1,0) \times t)\right\}=\left\{(1,0) ;\left(\zeta^{s}, 0\right): s \in I(k)\right\},
$$

which we show are transversal in the next section.

The group of deck transformations is $\mathbb{Z}_{p} \times \mathbb{Z}_{p}$, and the element $\left(\zeta^{j_{1}}, \zeta^{j_{2}}\right)$ acts on the Lefshetz dual homology representatives $A_{k}$ by

$$
\begin{array}{rlrl}
\left(\zeta^{j_{1}}, \zeta^{j_{2}}\right)\left(A_{k}\right) & =\left\{\left(\zeta_{q}^{j_{1}}, \zeta_{q}^{j_{2}}\right)\left(w, \zeta_{q}^{k-1+t} w\right)\right\} & & (\text { for } t \in I) \\
& =\left\{\left(\zeta_{q}^{j_{1}} w, \zeta_{q}^{k-1+t+j_{2}} w\right)\right\} & \\
& =\left\{\left(w, \zeta_{q}^{k-1+t+\left(j_{2}-j_{1}\right)} w\right)\right\} & & \text { (as subspaces) } \\
& =A_{k+\left(j_{2}-j_{1}\right)} & &
\end{array}
$$

Thus the diagonal subgroup of $\mathbb{Z}_{p} \times \mathbb{Z}_{p}$ acts trivially. We restrict our attention to the action by the subgroup generated by $(1, \zeta)$, which is isomorphic to the quotient of $\mathbb{Z}_{p} \times \mathbb{Z}_{p}$ by this kernel. Notice also that $\left(\zeta_{q}^{j_{1}}, \zeta_{q}^{j_{2}}\right)(1, z)=\left(\zeta_{q}^{j_{1}} 1, \zeta^{j_{2}} z\right)$, thereby sending $S$ to $S^{\prime}$ another representative of $[S]$ that differs only by the choice of the point in the first coordinate. We deduce that $(1, \zeta)\left[A_{k} \cap S\right]=\left[A_{k+1} \cap S\right]$.

\subsection{The Heegard Decomposition}

When computing the intersections of these homology representatives in the next section, it will be useful to employ the canonical Heegard decomposition. To this end we define $T$ to be the solid torus $D^{2} \times[0, p]$ modulo the relation $(x, 0) \sim(x, p)$. We describe points in $T$ using polar coordinates as $\left\{\left(r, \theta_{1}, \theta_{2}\right): r \in[0,1]\right.$ and $\left.\theta_{j} \in[0, p]\right\}$. Along the circle given by $r=0, \theta_{1}$ is irrelevant, so we denote such points as $\left(0,-, \theta_{2}\right)$, and refer to them as the central fiber of the torus. Let $T_{1}$ and $T_{2}$ to be two copies of $T$ and set $F: \partial T_{1} \longrightarrow \partial T_{2}$, by $\left(1, \theta_{1}, \theta_{2}\right) \longmapsto\left(1, \theta_{2}, \theta_{1}\right)$. We have that $S^{3}=T_{1} \cup_{F} T_{2}$ and

$$
\widetilde{\operatorname{Conf}}_{2}(L(p, q)) \subset\left(T_{1} \cup_{F} T_{2}\right) \times\left(T_{3} \cup_{F} T_{4}\right) .
$$

When using this decomposition to define a map we use superscripts to indicate which torus we are mapping to. The action of $\zeta_{q}$ in the torus decomposition of $S^{3}$ is 
described by

$$
\begin{aligned}
& \zeta_{q}\left(r_{1}, \theta_{2}, \theta_{1}\right)=\left(r_{1}, \theta_{2}+q, \theta_{1}+1\right) \\
& \zeta_{q}\left(r_{2}, \theta_{1}, \theta_{2}\right)=\left(r_{2}, \theta_{1}+1, \theta_{2}+q\right) .
\end{aligned}
$$

Here the first equation describes the action in $T_{1}$ and the second in $T_{2}$. Furthermore, we note that when using the torus decomposition of $S^{3}$ we can write $A_{k}$ as the union $\Gamma\left(\alpha_{k}^{1}\right) \cup \Gamma\left(\alpha_{k}^{2}\right)$ where

$$
\begin{aligned}
\alpha_{k}^{1}: T \times I(k) & \longrightarrow T \\
\left(r_{1}, \theta_{1}, \theta_{2}\right) \times s & \longmapsto\left(r_{1}, \theta_{1}+q s, \theta_{2}+s\right) \\
\alpha_{k}^{2}: T \times I(k) & \longrightarrow T \\
\left(r_{2}, \theta_{3}, \theta_{4}\right) \times s & \longmapsto\left(r_{2}, \theta_{3}+s, \theta_{4}+q s\right) .
\end{aligned}
$$

\section{Intersection theory and Massey products}

In this section we determine the Massey product structure for $\widetilde{\operatorname{Conf}}{ }_{2}(L(p, q))$. Let $S^{1}$ be $\mathbb{R}$ modulo the subgroup $p \mathbb{Z}$, and given an interval $(a, b) \subset \mathbb{R}$, let $(a, b)_{S^{1}}$ denote its projection on $S^{1}$. Many of the following computations depend on the intersection of specific intervals on $S^{1}$. These intervals depend on the choice of $q$, hence we make the following definition.

Definition 3.1. Let $j$ be an integer modulo $p$. Define $j$ to be $q$-covering if

$$
j \equiv q^{-1} m \quad(\bmod p) \quad \text { for }|m| \in(0, q)_{S^{1}} .
$$

Fix $j$ to be $q$-covering. Now define $k$ to be an interloper of $j$ if

$$
\begin{array}{ll}
k \in[j, p]_{S^{1}} \quad \text { and } & m>0, \text { or } \\
k \in[0, j]_{S^{1}} \text { and } & m<0 .
\end{array}
$$

If $j$ is not $q$-covering then there are no interlopers of $j$.

To conveniently summarize the results of this section, we use a model for the cochains of $\widetilde{\operatorname{Conf}}_{2}(L(p, q))$ defined in Section 4.

Theorem 3.2. All non-trivial Massey products in $\widetilde{\operatorname{Conf}}{ }_{2}(L(p, q))$ are determined by the action of $\mathbb{Z}_{p}$ on the $a_{i}$ 's, linearity and the following chain level products, with the choice of bounding chains stated in Definition 3.20 below:

$$
\begin{aligned}
& \left\langle a_{1+j}, a_{1}, a_{1+k}\right\rangle=\eta a_{1+k}, \text { for } k \text { a non-zero interloper of } j \text { but not } q \text {-covering } \\
& \left\langle a_{1+j}, a_{1}, a_{1+j^{\prime}}\right\rangle=\eta\left(a_{1+j^{\prime}}-a_{1+j}\right), \text { for } j \text { and } j^{\prime} \text { interlopers of each other } \\
& \left\langle a_{1+j}, a_{1}, a_{1}\right\rangle=\sum_{k} \eta a_{1+k}, \text { where the sum ranges over all non-zero interlopers of } j .
\end{aligned}
$$

Example 3.3. For the Lens space $L(7,2)$ we have the following non-trivial chain level Massey products,

$$
\begin{aligned}
\left\langle a_{4}, a_{1}, a_{2}\right\rangle & =\eta a_{2}, & \left\langle a_{5}, a_{1}, a_{6}\right\rangle & =\eta a_{6} \\
\left\langle a_{4}, a_{1}, a_{3}\right\rangle & =\eta a_{3}, & \left\langle a_{5}, a_{1}, a_{0}\right\rangle & =\eta a_{0} \\
\left\langle a_{4}, a_{1}, a_{1}\right\rangle & =\eta\left(a_{2}+a_{3}+a_{4}\right), & \left\langle a_{5}, a_{1}, a_{1}\right\rangle & =\eta\left(a_{5}+a_{6}+a_{0}\right) .
\end{aligned}
$$

The first Massey product here is the example constructed by Longoni and Salvatore 


$\begin{array}{cc}\frac{T_{\vec{w}}\left(A_{k}\right)}{(0,0) ;\left(i \zeta^{s}, 0\right)} & \frac{T_{\vec{w}}(S)}{(0,1) ;\left(0, \zeta^{q s}\right)} \\ (0, i) ;\left(0, i \zeta^{q s}\right) & (0,0) ;\left(i \zeta^{s}, 0\right) \\ (i, 0) ;\left(i \zeta^{s}, 0\right) & (0,0) ;\left(0, \zeta^{q s}\right) ;\left(0, i \zeta^{q s}\right)\end{array}$.

Table 2: The tangent vectors at a point $\vec{w} \in S \cap A_{k}$.

in $[\mathbf{8}]$, up to the indeterminacy.

We use intersection theory to compute these Massey (triple) products which we define at the chain level. Given a differential graded algebra $A$ and elements $u, v, x, y$, and $z \in A$ such that

$$
d x=d y=d z=0, \quad d u=x y, \quad \text { and } \quad d v=y z,
$$

we define the Massey product

$$
\langle x, y, z\rangle:=u z-(-1)^{|x|} x v .
$$

The notation $|x|$ denotes the degree of $x$.

Remark 3.4. This Massey product depends on choices of $u$ and $v$. So a better notation would be $\langle x, y, z\rangle_{(u, v)}$. But we will instead make our choices of $u$ and $v$ clear outside of the notation. When defined at the cohomology level, a Massey product $\langle[x],[y],[z]\rangle$ lives in the cohomology ring $H(A)$, modulo the ideal generated by $[x]$ and $[z]$. Thus in Example 3.3 all but the last two Massey products vanish at the cohomology level.

\subsection{Intersection Computations}

Some of the intersections among our representing submanifolds are transversal, and others are not. We describe all double and triple intersections among the representing manifolds $A_{k}$. When the intersections are not transversal we describe explicit perturbations that make them so. When an intersection is transversal, we sometimes denote it by $U \pitchfork V$.

Example 3.5. To give an example of a transversal intersection, we justify the claim from the previous section that $S \cap A_{k}$ is transversal. Recall that

$$
S \cap A_{k}=\left\{\Gamma\left(\alpha_{k}\right)((1,0) \times s): s \in I(k)\right\}=\left\{(1,0) ;\left(\zeta^{s}, 0\right): s \in I(k)\right\},
$$

and notice that is has the correct dimension. To show this intersection is transversal, we span the tangent space at a point

$$
\vec{w}=\left(w_{1}, w_{2}\right) \in \widetilde{\operatorname{Conf}}_{2}(L(p, q)) \subset S^{3} \times S^{3}
$$

using tangent vectors of $A_{k}$ and $S$. Consulting Definitions 2.2 and 2.3, and then finding perpendicular vectors in $\mathbb{C}^{2} \times \mathbb{C}^{2} \cong \mathbb{R}^{4} \times \mathbb{R}^{4}$ that are tangent to $S^{3} \times S^{3}$, we see that the tangent vectors of $\vec{w}$ are listed in Table 2 . These tangent vectors clearly span a six-dimensional space, since only the top two vectors are linearly dependent. 
We also remark that it is sufficient to restrict our attention to $q<\frac{p}{2}$. If $q$ were greater than $\frac{p}{2}$ we could replace our chosen representative $A_{k}$ with

$$
\breve{A}_{k}:=\left\{\left(z_{1}, z_{2}\right) ;\left(\zeta^{k-1+t} z_{1}, \zeta^{q(k-1)+(q-p) t)} z_{2}\right): t \in I\right\} .
$$

This yields similar results to those which we prove for $q<\frac{p}{2}$.

\subsubsection{Double Intersections}

We begin by computing the intersection of $A_{k}$ and $A_{k+j}$. Recall our chosen representatives for the the generators $\left[A_{k}\right] \in H_{4}\left(S^{3} \times S^{3}, \Delta_{q}\right)$ are $A_{k}=\Gamma\left(\alpha_{k}\right)$ as given in Definition 2.2. These representatives have codimension two so the transversal intersection of any two of these representatives should have codimension four. A transversal intersection of three of them is a collection of points, and any transversal intersection of more than three is empty.

In computing intersections we make use of the projections $p r_{i}: S^{3} \times S^{3} \longrightarrow S^{3}$ sending $\left(w_{1}, w_{2}\right)$ to $w_{i}$. If we consider the equality

$$
p r_{1} \Gamma\left(\alpha_{k}\right)(w, s)=p r_{1} \Gamma\left(\alpha_{k+j}\right)\left(w^{\prime}, s^{\prime}\right),
$$

where $s \in I(k)$ and $s^{\prime} \in I(k+j)$, we see that any point in $A_{k} \cap A_{k+j}$ would have $w$ equal $w^{\prime}$. Applying the second projection we see $\alpha_{k}(w)$ must equal $\alpha_{k+j}\left(w^{\prime}\right)$ as well, so we have

$$
\zeta_{q}^{s} w=\zeta_{q}^{s^{\prime}} w
$$

Thus, letting $w=\left(z_{1}, z_{2}\right)$ we have

$$
\begin{array}{cccc}
\zeta^{s} & =\zeta^{s^{\prime}} & \text { or } & z_{1}=0, \\
\zeta^{q s} & =\zeta^{q s^{\prime}} & \text { or } & z_{2}=0 .
\end{array}
$$

It is clear that unless $j=0$, Equation (1) only has solutions when $z_{1}=0$. So the intersection occurs along a central fiber. We assume $j \neq 0$ for now and discuss the case when $j=0$ in Section 3.1.3. So the intersection in question is of the form

$$
A_{k} \cap A_{k+j}=\left\{\Gamma\left(\alpha_{1}\right)\left(\left(0, z_{2}\right) \times \tau\right)\right\}
$$

for some $\tau$ which is determined by the solution to the equation (2). This equation can be written as

$$
q\left(s-s^{\prime}\right) \equiv 0 \quad(\bmod p) \quad \text { or } \quad q(j+\lambda) \equiv 0 \quad(\bmod p),
$$

where $\lambda \in(-1,1)$, so $q \lambda \in(-q, q)_{S^{1}}$. As $q j$ is a non-zero integer this equation has solutions exactly when $q j \equiv m(\bmod p)$ for $|m| \in(0, q)$.

Remark 3.6. The definition of $q$-covering in Definition 3.1 arises from this computation. If $j$ is not $q$-covering then $A_{k} \cap A_{k+j}$ is empty.

Remark 3.7. We adopt the notational convention that all $s$, including those that are decorated (for example $s^{\prime \prime}$ ), are in $I(k)$ for an appropriate choice of $k$. We reserve $t$, including those that are decorated, for quantities which vary in $I=(0,1)$. Lastly, we let $\tau \in I_{\tau}$, where $I_{\tau}$ is the interval $(q(\ell-1), q \ell)_{S^{1}} \cap\left(q\left(\ell^{\prime}-1\right), q \ell^{\prime}\right)_{S^{1}}$ for the appropriate choice of $\ell$ and $\ell^{\prime}$. This convention holds for decorated $\tau$ 's as well. Note that $I_{\tau}$ can be empty. 
Proposition 3.8. If $j$ is q-covering and $I_{\tau}=(q I(k))_{S^{1}} \cap(q I(k+j))_{S^{1}}$, then the intersection

$$
\begin{aligned}
A_{k} \cap A_{k+j} & =\left\{\Gamma\left(\alpha_{k}\right)\left(\left(0, z_{2}\right) \times \tau\right): \tau \in I_{\tau}\right\} \\
& =\left\{\left(0, z_{2}\right) ;\left(0, \zeta^{\tau} z_{2}\right): \tau \in I_{\tau}\right\}
\end{aligned}
$$

is non-empty, transversal and is homeomorphic to $S^{1} \times I_{\tau}$.

Proof. Our previous discussion establishes that such an intersection is non-empty. To show the intersection is transversal we let $s \in I(k)$ and $s^{\prime} \in I(k+j)$ and record the tangent vectors in $\mathbb{C}^{2} \times \mathbb{C}^{2}$ as follows:

$$
\begin{array}{cc}
\frac{T_{\vec{w}}\left(A_{k}\right)}{(1,0) ;\left(\zeta^{s}, 0\right)} & \frac{T_{\vec{w}}\left(A_{k+j}\right)}{(1,0) ;\left(\zeta^{s^{\prime}}, 0\right)} \\
(i, 0) ;\left(i \zeta^{s}, 0\right) & (i, 0) ;\left(i \zeta^{s^{\prime}}, 0\right) \\
(0, i) ;\left(0, i \zeta^{q s} z_{2}\right) & (0, i) ;\left(0, i \zeta^{q s^{\prime}} z_{2}\right) \\
(0,0) ;\left(0, i \zeta^{q s} z_{2}\right) & (0,0) ;\left(0, i \zeta^{q s^{\prime}} z_{2}\right)
\end{array} .
$$

Since we are evaluating these tangent vectors at a point where $\zeta^{q s}=\zeta^{q s^{\prime}}$, the last two pairs of tangent vectors are linearly dependent and thus span a two dimensional space. The remaining four are linearly independent since $j \neq 0$. Thus these span a six-dimensional space showing that the intersection is transversal.

Example 3.9. If we consider $p=11$ and $q=3$, this proposition says that for $A_{k} \cap A_{k+j}$ to be non-empty $j \equiv 4 m(\bmod p)$ for $m=-2,-1,1,2$, so $j=3,7,4,8$.

Remark 3.10. Many of the theorems in this section follow from straightforward though tedious computations. We omit several of these computations, noting that the structure is similar to the proof of Proposition 3.8. Namely, we would proceed to show that an intersection must occur on the central fiber of a torus, and then use number theory to determine the exact intersection. This kind of argument works even with perturbations, though more care is needed. For the interested reader the computations are given in the author's thesis $[\mathbf{9}]$.

\subsubsection{Triple Intersections}

We saw above that all of the double intersections of the $A_{k}$ 's are transversal. This is not the case with the triple intersections. But in order to compute Massey products we need to calculate $A_{k} \cap X_{i j}$ where the boundary of $X_{i j}$ is $A_{i} \cap A_{j}$. The boundary $A_{k} \cap \partial X_{i j}$ is $A_{k} \cap A_{i} \cap A_{j}$, so we begin by understanding all of the triple intersections of distinct $A_{k}$. By the action of $\mathbb{Z}_{p}=\langle(1, \zeta)\rangle$ described in Section 2.1, it suffices to consider $i=1$.

From the previous section we know that when $j$ and $j^{\prime}$ are $q$-covering, and $I_{\tau}$ and $I_{\tau^{\prime}}$ are as described in Remark 3.7,

$$
\begin{aligned}
& A_{1} \cap A_{1+j}=\left\{\left(0, z_{2}\right) ;\left(0, \zeta^{\tau} z_{2}\right): \tau \in I_{\tau}\right\} \\
& A_{1} \cap A_{1+j^{\prime}}=\left\{\left(0, z_{2}^{\prime}\right) ;\left(0, \zeta^{\tau^{\prime}} z_{2}^{\prime}\right): \tau^{\prime} \in I_{\tau^{\prime}}\right\} .
\end{aligned}
$$

So the only non-trivial triple intersections of distinct $A_{k}$ must be of the form

$$
A_{1} \cap A_{1+j} \cap A_{1+j^{\prime}}=\left\{\left(0, z_{2}\right) ;\left(0, \zeta^{\tau} z_{2}\right): \tau \in I_{\tau} \cap I_{\tau^{\prime}}\right\} .
$$

If this is non-empty, it is not transversal because its dimension is two, not zero. 
That there are non-empty intersections of this form now follows from an elementary calculation.

Proposition 3.11. Let $j$ and $j^{\prime}$ be q-covering so $j \equiv m q^{-1}$, and $j^{\prime} \equiv m^{\prime} q^{-1}$ with $|m|,\left|m^{\prime}\right| \in(0, q)_{S^{1}}$. Then the triple intersection $A_{1} \cap A_{1+j} \cap A_{1+j^{\prime}}$ is non-empty if and only if $\left|m-m^{\prime}\right|$ is in $(0, q)_{S^{1}}$.

Example 3.12. When, $p=11$ and $q=3$ the only non-empty triple intersections of distinct $A_{k}$ 's are $A_{k} \cap A_{k+7} \cap A_{k+4}$.

The main result in this section resolves these non-transversal triple intersections.

Theorem 3.13. There exist arbitrarily small perturbations of $A_{1+j^{\prime}}$, denoted by $\widetilde{A}_{1+j^{\prime}}$ and defined below, so that the intersection $A_{1} \cap A_{1+j} \cap \widetilde{A}_{1+j^{\prime}}$ is empty and hence transversal.

First recall that $A_{k}$ is the track of $\alpha_{k}$, which in the torus decomposition is the track of $\alpha_{k}^{1} \cup \alpha_{k}^{2}$. The double intersections and the non-transversal triple intersections are contained in the central fiber and thus in $\Gamma\left(\alpha_{k}^{2}\right)\left(\left(0,-, \theta_{2}\right) \times s\right)$. Since our perturbations will be arbitrarily small and these intersections are at the center of the tori, we need only consider the image of $\alpha_{k}^{2}$.

In order to more easily perturb the representatives we use rectangular coordinates on the range of our map $\alpha_{1+j^{\prime}}^{2}: T \times I\left(j^{\prime}\right) \rightarrow T$ defined as

$$
\alpha_{1+j^{\prime}}^{2}:\left(r, \theta_{1}, \theta_{2}\right) \times s^{\prime} \longmapsto\left(r \cos \left(\frac{2 \pi}{p}\left(\theta_{1}+s^{\prime}\right)\right), r \sin \left(\frac{2 \pi}{p}\left(\theta_{1}+s^{\prime}\right)\right), \theta_{2}+q s^{\prime}\right) .
$$

There are two functions needed to perturb $\alpha_{1+j^{\prime}}^{2}$. The first function depends on $t^{\prime}=s^{\prime}-j^{\prime} \in I$. Let

$$
f\left(t^{\prime}\right)= \begin{cases}t^{\prime}, & t^{\prime} \in\left(0, \frac{1}{2}\right] \\ 1-t^{\prime}, & t^{\prime} \in\left[\frac{1}{2}, 1\right) .\end{cases}
$$

The next function will be used to alter $\alpha_{1+j^{\prime}}^{2}$ according to the distance from the central fiber of the tori. Let

$$
g(r)= \begin{cases}1, & r \in\left[0, \frac{1}{4}\right] \\ 2-4 r, & r \in\left[\frac{1}{4}, \frac{1}{2}\right] \\ 0, & r \in\left[\frac{1}{2}, 1\right] .\end{cases}
$$

Definition 3.14. Let $\widetilde{A}_{1+j^{\prime}}$ be the union $\Gamma\left(\alpha_{1+j^{\prime}}^{1}\right) \cup \Gamma\left(\widetilde{\alpha}_{1+j^{\prime}}^{2}\right)$, where

$$
\begin{gathered}
\widetilde{\alpha}_{1+j^{\prime}}^{2}: T \times I\left(1+j^{\prime}\right) \longrightarrow T_{2} \times T_{4}, \\
\widetilde{\alpha}_{1+j^{\prime}}^{2}:\left(r, \theta_{1}, \theta_{2}\right) \times s^{\prime} \longmapsto \\
\left(r \cos \left(\frac{2 \pi}{p}\left(\theta_{1}+s^{\prime}\right)\right), r \sin \left(\frac{2 \pi}{p}\left(\theta_{1}+s^{\prime}\right)\right)+f\left(t^{\prime}\right) g(r) \varepsilon, \theta_{2}+q s^{\prime}\right),
\end{gathered}
$$

where $s^{\prime} \in I\left(1+j^{\prime}\right), t^{\prime}=s^{\prime}-j^{\prime}$ and $\varepsilon>0$ is constant and close to zero.

This perturbation shifts the $y$ coordinate of $\widetilde{\alpha}_{1,1+j^{\prime}}^{2}$ by an arbitrarily small amount which goes to zero as points approach the boundary of the torus, $\Delta_{q}^{j^{\prime}}$ and $\Delta_{q}^{1+j^{\prime}}$. In 
Figure 1 we illustrate the image of $\alpha_{1+j^{\prime}}^{2}\left(x \times I\left(1+j^{\prime}\right)\right)$ and $\alpha_{1+j^{\prime}}^{2}\left(y \times I\left(1+j^{\prime}\right)\right)$ by dotted lines and their perturbations $\widetilde{\alpha}_{1+j^{\prime}}^{2}\left(x \times I\left(1+j^{\prime}\right)\right)$ and $\widetilde{\alpha}_{1+j^{\prime}}^{2}\left(y \times I\left(1+j^{\prime}\right)\right)$ by solid lines.

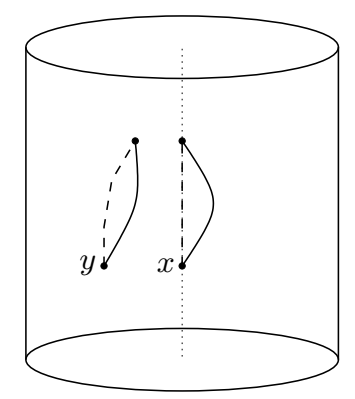

Figure 1: Some "fibers" in $A_{1+j^{\prime}}$ and its perturbation $\widetilde{A}_{1+j^{\prime}}$

Proof. (of Theorem 3.13)

Recall that $A_{1} \cap A_{1+j} \subset \Gamma\left(\alpha_{1}^{2}\right)\left(\left(0,-, \theta_{2}\right) \times t\right)$, which is in the central fiber of $T_{2} \times T_{4}$. We show that $A_{1} \cap \widetilde{A}_{1+j^{\prime}}$ does not intersect $\Gamma\left(\alpha_{1}^{2}\right)\left(\left(0,-, \phi_{2}\right) \times s^{\prime}\right)$. Consider $A_{1} \cap \widetilde{A}_{1+j^{\prime}}$, our perturbation does not effect the projection onto the first coordinate, so $\operatorname{pr}_{1} \Gamma\left(\alpha_{1+j^{\prime}}\right)=p r_{1} \Gamma\left(\widetilde{\alpha}_{1+j^{\prime}}\right)$ implying that $\alpha_{1}(w, s)=\widetilde{\alpha}_{1+j^{\prime}}\left(w^{\prime}, s^{\prime}\right)$ only when $w=w^{\prime}$. Now we consider the equality arising from the projection onto the second coordinate, namely $\alpha_{1}\left(\left(0,-, \theta_{2}\right) \times t\right)=\widetilde{\alpha}_{1+j^{\prime}}\left(\left(0,-, \theta_{2}\right) \times s^{\prime}\right)$. Using the definitions of $\alpha_{1}, \widetilde{\alpha}_{1+j^{\prime}}$ and $g(r)$ we see that

$$
\begin{aligned}
\Gamma\left(\alpha_{1}\right)\left(\left(0,-, \theta_{2}\right) \times t\right) & =\left(0,0, \theta_{2}\right) \times\left(0,0, \theta_{2}+q t\right) \\
\Gamma\left(\widetilde{\alpha}_{1+j^{\prime}}\right)\left(\left(0,-, \theta_{2}\right) \times s^{\prime}\right) & =\left(0,0, \theta_{2}\right) \times\left(0, f\left(t^{\prime}\right) \varepsilon, \theta_{2}+q s^{\prime}\right),
\end{aligned}
$$

where $t^{\prime}=s^{\prime}-j^{\prime}$. Because $f\left(t^{\prime}\right) \varepsilon$ is never zero,

$$
\Gamma\left(\alpha_{1}\right)\left(\left(0,-, \theta_{2}\right) \times t\right) \cap \Gamma\left(\alpha_{1+j^{\prime}}\right)\left(\left(0,-, \phi_{2}\right) \times s^{\prime}\right)
$$

is empty, which finishes the proof of the theorem.

When we compute Massey products we will need to understand $A_{1} \cap \widetilde{A}_{1+j^{\prime}}$ explicitly. As we have all the definitions at hand we present this intersection now. This intersection is a small perturbation of $A_{1} \cap A_{1+j^{\prime}}$ and thus occurs close to the central fiber of the second torus. Heuristically, this is the original intersection "pushed off the central fiber in one direction".

Proposition 3.15. Let $\tau \in I_{\tau}$, as defined in Remark 3.7, let $\square=\frac{p}{2}-\frac{1}{2}\left(j^{\prime}-\frac{m^{\prime}}{q}\right)$, and let

$$
r(t)=\frac{f\left(t-\frac{m}{q}\right) \varepsilon}{2 \sin \left(\frac{2 \pi}{p} \square\right)} .
$$

Then we have the following intersection

$$
A_{1} \cap \widetilde{A}_{1,1+j^{\prime}}=\Gamma\left(\alpha_{1}^{2}\left(\left(r(t), \square, \theta_{2}\right) \times \tau\right)\right) .
$$


We note that with an appropriate choice of $\varepsilon$ the intersection $A_{1} \cap \widetilde{A}_{1+j^{\prime}}$ occurs arbitrarily close to the central fiber.

\subsubsection{Self Intersections}

The self intersection $A_{1} \cap A_{1}$, which is clearly not transversal, plays an important role in the Massey product structure of $\widetilde{\operatorname{Conf}}{ }_{2}(L(p, q))$. For the purpose of this selfintersection we use a perturbation of $A_{1}$ that is slightly different than the perturbation in the previous section. We need another function which changes the perturbation according to the distance from the center of the tori,

$$
h(r)= \begin{cases}0, & r \in\left[0, \frac{1}{4}\right] \\ 4 r-1, & r \in\left[\frac{1}{4}, \frac{1}{2}\right] \\ 1, & r \in\left[\frac{1}{2}, 1\right] .\end{cases}
$$

We now define a new perturbation of $A_{1} \cong S^{3} \times(0,1)$ using rectangular coordinates that rescales the interval and the distance from the central fiber, as well as shifts the $y$ coordinate.

Definition 3.16. Let $\widehat{A}_{1}$ be $\Gamma\left(\widehat{\alpha}_{1}\right)$, the track of the map

$$
\begin{gathered}
\widehat{\alpha}_{1}=\widehat{\alpha}_{1}^{1} \cup \widehat{\alpha}_{1}^{2}: S^{3} \times I \longrightarrow S^{3} \\
\widehat{\alpha}_{1}^{1}:\left(r_{1}, \theta_{1}, \theta_{2}\right) \times t \longmapsto\left(\left(1-f(t) h\left(r_{1}\right) \varepsilon_{3}\right) r_{1} \cos \left(\frac{2 \pi}{p}\left(\theta_{1}+q\left(t+f(t) g\left(r_{1}\right) \varepsilon_{1}\right)\right)\right),\right. \\
\left.\left(1-f(t) h\left(r_{1}\right) \varepsilon_{3}\right) r_{1} \sin \left(\frac{2 \pi}{p}\left(\theta_{1}+q\left(t+f(t) g\left(r_{1}\right) \varepsilon_{1}\right)\right)\right)+f(t)^{2} g\left(r_{1}\right) \varepsilon_{2}, \theta_{2}+t\right) \\
\widehat{\alpha}_{1}^{2}:\left(r_{2}, \theta_{3}, \theta_{4}\right) \times t \longmapsto\left(\left(1+f(t) h\left(r_{2}\right) \varepsilon_{3}\right) r_{2} \cos \left(\frac{2 \pi}{p}\left(\theta_{3}+t+f(t) g\left(r_{2}\right) \varepsilon_{1}\right)\right),\right. \\
\left.\left(1+f(t) h\left(r_{2}\right) \varepsilon_{3}\right) r_{2} \sin \left(\frac{2 \pi}{p}\left(\theta_{3}+t+f(t) g\left(r_{2}\right) \varepsilon_{1}\right)\right)+f(t)^{2} g\left(r_{2}\right) \varepsilon_{2}, \theta_{4}+q t\right) .
\end{gathered}
$$

Here we let $\varepsilon_{1}>0, \varepsilon_{2}>0$, and $\varepsilon_{3}>0$ be fixed.

\section{Remark 3.17.}

- Away from the central fibers, the radius has been contracted in the first torus and dilated in the second torus, so the radius is multiplied by $1-f(t) h\left(r_{2}\right) \varepsilon_{3}$ for $\alpha_{1}$ and $1+f(t) h\left(r_{2}\right) \varepsilon_{3}$ for $\alpha_{2}$. If this were the only perturbation the intersection $\Gamma\left(\alpha_{1}\right) \cap \Gamma\left(\widehat{\alpha}_{1}\right)$ would occur on all of the subspace defined by $r_{i} \leqslant \frac{1}{4}$.

- The perturbation in the inputs of sine and cosine ensures that for a given point in the torus and a fixed $t=t^{\prime}$, the image under $\alpha$ is different than the image under $\widehat{\alpha}$. With only these first two perturbations, the intersection $\Gamma\left(\alpha_{1}\right) \cap \Gamma\left(\widehat{\alpha}_{1}\right)$ would occur on the central fiber, which has the correct dimension but is not transversal.

- The shift in the $y$ coordinates is the same as the perturbation in the previous section. This factor pushes the intersection off of the central fiber, and makes the intersection transversal. We square $f(t)$ so that this shift is close to zero when $t$ is close to 0 and 1 . 
Theorem 3.18. The intersection $A_{1} \cap \widehat{A}_{1}$ is a transversal perturbation of the self intersection $A_{1} \cap A_{1}$ and is equal to

$$
A_{1} \cap \widehat{A}_{1}=\Gamma\left(\alpha_{1}^{1}\right)\left(\left(r_{1}(t), \square_{1}, \theta_{2}\right) \times t\right) \cup \Gamma\left(\alpha_{1}^{2}\right)\left(\left(r_{2}(t), \square_{2}, \theta_{4}\right) \times t\right)
$$

for functions

$$
\begin{aligned}
r_{1}(t) & =\frac{f(t)^{2} \varepsilon}{2 \sin \left(\frac{2 \pi}{p}\left(\square_{1}+q t\right)\right)} \\
r_{2}(t) & =\frac{f(t)^{2} \varepsilon}{2 \sin \left(\frac{2 \pi}{p}\left(\square_{2}+t\right)\right)} \\
\square_{1} & =\frac{p}{2}-\frac{q}{2} f(t) \varepsilon-q t \\
\square_{2} & =\frac{p}{2}-\frac{1}{2} f(t) \varepsilon-t .
\end{aligned}
$$

Moreover, with an appropriate choice of $\varepsilon$ this intersection lies arbitrarily close to the central fiber.

In this intersection there are two components because, unlike in previous cases, there is a non-trivial intersection of $A_{1}$ with itself along the central fiber of the first torus. We also note that the functions $r_{i}(t)$ are similar to those in Proposition 3.15.

Proposition 3.19. The triple intersection $A_{1} \cap \widehat{A}_{1} \cap A_{j}$ is empty

Proof. This proposition follows immediately from the fact that $A_{1} \cap A_{1+j}$ is empty unless $j$ is $q$-covering, in which case it occurs on the central fiber of $T_{2} \times T_{4}$. But $A_{1} \cap \widehat{A}_{1}$ does not intersect the central fiber, as stated in Theorem 3.18.

\subsection{Massey Products}

The Massey product $\langle x, y, z\rangle$ is in degree $|x|+|y|+|z|-1$. Therefore a non-trivial Massey product in $C^{*}\left(\widetilde{\operatorname{Conf}}_{2}(L(p, q))\right)$ must involve cycles $x, y, z$ all in degree 2. Moreover, at the chain level Massey products are linear, so we need only consider when these cycles are generators.

In order to compute Massey products of the form $\left\langle a_{i}, a_{j}, a_{k}\right\rangle$ it is necessary to find bounding manifolds for the double intersections described above. The triple intersection will be relevant when intersecting the bounding manifolds with other $A_{k}$. Since all products of the form $\left[a_{k}\right] \cdot\left[a_{j}\right]$ are zero in the cohomology ring, there are bounding manifolds for all of the double intersections. We define three dimensional manifolds $X_{1,1+j}$ that have relative boundary equal to $A_{1} \cap A_{1+j}$.

Recall that $A_{1} \cap A_{1+j}$ is defined by an embedding of $S^{1} \times(0,1)$ along the central fiber of $T_{2} \times T_{4}$. The bounding manifolds $X_{1,1+j}$ are defined by an embedding of $D^{2} \times(0,1)$ so that the boundary $S^{1} \times(0,1)$ coincides with $A_{1} \cap A_{1+j}$ and the center of the disc is on the central fiber of $T_{1} \times T_{3}$. To be precise we identify $D^{2}$ with the image of the embedding

$$
\begin{aligned}
\delta: D^{2} & \longrightarrow T_{1} \cup_{F} T_{2}=S^{3} \\
\delta^{1}:(r, \theta) & \longmapsto\left(r_{1}, \theta, 0\right) \\
\delta^{2}:(r, \theta) & \longmapsto\left(r_{2}, 0, \theta\right),
\end{aligned}
$$

where $r_{1}=2 r$ for $r \in\left[0, \frac{1}{2}\right]$, and $r_{2}=2-2 r$ for $\left[\frac{1}{2}, 1\right]$. 
Definition 3.20. For $j q$-covering, define a three dimensional sub-manifold $X_{1,1+j}$ to be $\Gamma\left(\chi_{1,1+j}\right)$, the track of the map

$$
\begin{aligned}
\chi_{1,1+j}=\chi_{1,1+j}^{1} \cup \chi_{1,1+j}^{2}: D^{2} \times I_{\tau} \longrightarrow S^{3} \\
\chi_{1,1+j}^{1}:(r, \theta) \times \tau \longmapsto\left(r_{1}, \theta+\tau, \sigma\right) \\
\chi_{1,1+j}^{2}:(r, \theta) \times \tau \longmapsto\left(r_{2}, \sigma, \theta+\tau\right) .
\end{aligned}
$$

Here $r_{1}$ and $r_{2}$ are as described in the definition of $\delta, I_{\tau}=(0, q)_{S^{1}} \cap(m, m+q)_{S^{1}}$, $q \sigma \equiv \tau(\bmod p)$. If $m$ is negative then $\sigma \in(0, j)_{S^{1}}$, and if $m$ is positive then $\sigma \in(j, 0)_{S^{1}}$. We denote the interval of possible values for $\sigma$ by $I_{\sigma}$.

Alternately, in the complex model we have

$$
X_{1,1+j}=\left\{(\rho, z) ;\left(\rho \zeta^{\sigma}, \zeta^{\tau} z\right):|z|^{2}+\rho^{2}=1, \text { and } \rho \in \mathbb{R}\right\} .
$$

Since $A_{1} \cap A_{\ell}$ is empty when $\ell \neq 1+j$, where $j$ is $q$-covering, we set $X_{1, \ell}=\emptyset$ in this case.

Proposition 3.21. The manifold $X_{1,1+j}$ has boundary $A_{1} \pitchfork A_{1+j}$.

Proof. Since $\tau$ is in an open interval the only boundary occurs when $r=1$. But when $r=1$ we have $A_{1} \pitchfork A_{1+j}$.

The bounding manifold $\widehat{X}_{1,1}$ for the self intersection $A_{1} \cap \widehat{A}_{1}$ has two components. Both components are similar in structure to $X_{1,1+j}$ in that they are each an embedding of $D^{2} \times(0,1)$ such that the boundary circle coincides with a component of $A_{1} \cap \widehat{A}_{1}$ and the center of the disc lies on a central fiber. Recall from Theorem 3.18 that

$$
\begin{aligned}
& \square_{1}:=\frac{p}{2}-\frac{q}{2} f(t) \varepsilon-q t \\
& \square_{2}:=\frac{p}{2}-\frac{1}{2} f(t) \varepsilon-t
\end{aligned}
$$

and the functions $r_{i}(t)$ can be set arbitrarily close to zero with the choice of $\varepsilon$.

Definition 3.22. Let $\rho_{i}$ be in the interval $[0,1]$, for $i=1,2$ and define $\widehat{X}_{1,1}$ to be the union of $\Gamma\left(\beta_{1}\right)$ defined here and $\Gamma\left(\gamma_{1}\right)$ defined below in Definition 3.24.

$$
\begin{gathered}
\beta_{1}=\beta_{1}^{1} \cup \beta_{1}^{2}: D^{2} \times(0,1) \longrightarrow S^{3} \\
\beta_{1}^{1}:\left(\rho_{1}, \theta_{1}\right) \times t \longmapsto\left(\left(\rho_{1}+\left(1-\rho_{1}\right) r_{1}(t)\right) \cos \left(\frac{2 \pi}{p}\left(\left(1-\rho_{1}\right) \square_{1}+q t\right)\right)\right), \\
\left.\left.\left(\rho_{1}+\left(1-\rho_{1}\right) r_{1}(t)\right) \sin \left(\frac{2 \pi}{p}\left(\left(1-\rho_{1}\right) \square_{1}+q t\right)\right)\right), \theta_{1}+t\right) \\
\beta_{1}^{2}:\left(\rho_{2}, \theta_{3}\right) \times t \longmapsto\left(\rho_{2}, \theta_{3}+t, q t\right),
\end{gathered}
$$

where $\rho_{1}=2 \rho$ for $\rho \in\left[0, \frac{1}{2}\right], \rho_{2}=2-2 \rho$ for $\rho \in\left[\frac{1}{2}, 1\right]$.

Remark 3.23. If $r_{i}$ goes to zero, $\widehat{X}_{1,1}$ approaches a manifold whose boundary is on central fiber. The factors of $1-\rho$ are used to interpolate so that there is "no perturbation" on the boundary of the torus, which allows the halves to match up there. 
Definition 3.24. Similarly,

$$
\begin{gathered}
\gamma_{1}=\gamma_{1}^{1} \cup \gamma_{1}^{2}: D^{2} \times(0,1) \longrightarrow S^{3} \\
\gamma_{1}^{1}:\left(\rho_{1}, \theta_{2}\right) \times t \longmapsto\left(\rho_{1}, \theta_{2}+q t, t\right) \\
\gamma_{1}^{2}:\left(\rho_{2}, \theta_{4}\right) \times t \longmapsto\left(\left(\left(1-\rho_{2}\right) r_{2}(t)+\rho_{2}\right) \cos \left(\frac{2 \pi}{p}\left(\left(1-\rho_{2}\right) \square_{2}+t\right)\right)\right), \\
\left.\left.\quad\left(\left(1-\rho_{2}\right) r_{2}(t)+\rho_{2}\right) \sin \left(\frac{2 \pi}{p}\left(\left(1-\rho_{2}\right) \square_{2}+t\right)\right)\right), \theta_{4}+q t\right),
\end{gathered}
$$

where $\rho_{1}=2-2 \rho$ for $\rho \in\left[0, \frac{1}{2}\right], \rho_{2}=2 \rho$ for $\rho \in\left[\frac{1}{2}, 1\right]$.

Proposition 3.25. The manifold $\widehat{X}_{1,1}$ has boundary $A_{1} \cap \widehat{A}_{1}$.

Proof. The only boundary of $\widehat{X}_{1,1}$ occurs as the image of $\beta_{1}^{1}\left(0, \theta_{1}\right) \times t$ and $\gamma_{1}^{2}\left(0, \theta_{4}\right) \times$ $t$, but this is exactly $A_{1} \cap \widehat{A}_{1}$.

Remark 3.26. We note that $\left(1-\rho_{i}\right) r_{i}(t)+\rho_{i} \cdot 1$ is never zero since $r_{i}(t)$ is always positive. This implies that each component of $\widehat{X}_{1,1}$ only intersects one central fiber, and that is at the center of the disc. Schematically each component is similar to the perturbation pictured in Figure 2 below.

Massey products are represented by the submanifolds $\left(A_{\ell} \pitchfork X_{1, \ell^{\prime}}\right) \cup\left(A_{\ell^{\prime}} \pitchfork X_{1, \ell}\right)$, so we compute $A_{\ell} \pitchfork X_{1, \ell^{\prime}}$. In order to enumerate and explain the different cases more clearly we reset the notation from Definition 3.1. In particular, for the rest of this section we assume $j$ and $j^{\prime}$ are $q$-covering and $k$ and $k^{\prime}$ are interlopers for $j$ and $j^{\prime}$ respectively. We let $\ell$ be an arbitrary value in $\mathbb{Z}_{p}$. We need to calculate $A_{*} \pitchfork X_{1, \bullet}$ and $A_{*} \cap \widehat{X}_{1,1}$. There are several cases, and we establish a proposition for each case. Recall the definitions of $A_{\ell}$ and $X_{1,1+j}$ from Definitions 2.2 and 3.20.

Proposition 3.27. Let $j$ be q-covering as in Definition 3.1, and let $\ell \in \mathbb{Z}_{p}$. If $\ell$ is also q-covering assume that $|m-q \ell| \notin(0, q)_{S^{1}}$. Then

$$
A_{1+\ell} \cap X_{1,1+j}= \begin{cases}\emptyset, & \text { if } \ell \text { is not an interloper of } j \\ A_{1+\ell} \cap S, & \text { if } \ell \text { is an interloper of } j .\end{cases}
$$

Moreover, in the second case the induced orientations on $A_{\ell} \cap X_{1,1+j}$ and $A_{\ell} \cap S$ agree.

Next, the case when $|m-q \ell| \in(0, q)_{S^{1}}$. When $j$ and $j^{\prime}$ are both $q$-covering with $\left|m-m^{\prime}\right| \in(0, q)_{S^{1}}$ the intersection $A_{1+j^{\prime}} \cap A_{1} \cap A_{1+j}$ is not transversal, so neither is $A_{1+j^{\prime}} \cap X_{1,1+j}$. Thus it requires a perturbation like those described in Section 3.1. We define $\widetilde{X}_{1,1+j}$ to be a bounding manifold for $A_{1} \cap \widetilde{A}_{1+j}$ using a perturbed embedding of $D^{2} \times(0,1)$. Recall that $\square=\frac{p}{2}-\frac{1}{2}\left(j-\frac{m}{q}\right)$ and $r(t)$ is close to zero and is defined by Equation (3).

For $\tau \in I_{\tau}, q \sigma=\tau$ and $\sigma \in I_{\sigma}$ as in Remark 3.7 we perturb the first twisting factor with the function.

$$
\sigma_{\tau}:= \begin{cases}\sigma+\left(\tau-\inf \left\{I_{\tau}\right\}\right) \varepsilon, & \tau \in\left(\inf \left\{I_{\tau}\right\}, \frac{1}{2}\left(\sup \left\{I_{\tau}\right\}-\inf \left\{I_{\tau}\right\}\right)\right) \\ \sigma+\left(\sup \left\{I_{\tau}\right\}-\tau\right) \varepsilon, & \tau \in\left(\frac{1}{2}\left(\sup \left\{I_{\tau}\right\}-\inf \left\{I_{\tau}\right\}\right), \sup \left\{I_{\tau}\right\}\right) .\end{cases}
$$


Definition 3.28. Define $\widetilde{X}_{1,1+j}$ to be $\Gamma\left(\widetilde{\chi}_{1,1+j}\right)$, where

$$
\begin{aligned}
& \tilde{\chi}_{1,1+j}= \tilde{\chi}_{1,1+j}^{1} \cup \tilde{\chi}_{1,1+j}^{2}: D^{2} \times I \longrightarrow S^{3} \\
& \tilde{\chi}_{1,1+j}^{1}:(\rho, \theta) \times \tau \longmapsto\left(\rho_{1}, \theta+\tau, \sigma_{\tau}\right) \\
& \tilde{\chi}_{1,1+j}^{2}:(\rho, \theta) \longmapsto\left(( \rho _ { 2 } + ( 1 - \rho _ { 2 } ) r ( \tau ) ) \operatorname { c o s } \left(\frac{2 \pi}{p}\left(\left(1-\rho_{2}\right) \square+\sigma_{\tau}\right),\right.\right. \\
& \quad\left(\left(1-\rho_{2}\right) r(\tau)+\rho_{2}\right) \sin \left(\frac{2 \pi}{p}\left(\left(1-\rho_{2}\right) \square+\sigma_{\tau}\right), \theta+\tau\right),
\end{aligned}
$$

and where, $\rho_{1}=2 \rho$ for $\rho \in\left[0, \frac{1}{2}\right]$ and $\rho_{2}=2-2 \rho$ for $\rho \in\left[\frac{1}{2}, 1\right]$.

We illustrate $p r_{1} \Gamma\left(\chi_{1,1+j^{\prime}}\right)$ on the left and $p r_{1} \Gamma\left(\widetilde{\chi}_{1,1+j^{\prime}}\right)$ on the right of Figure 2.
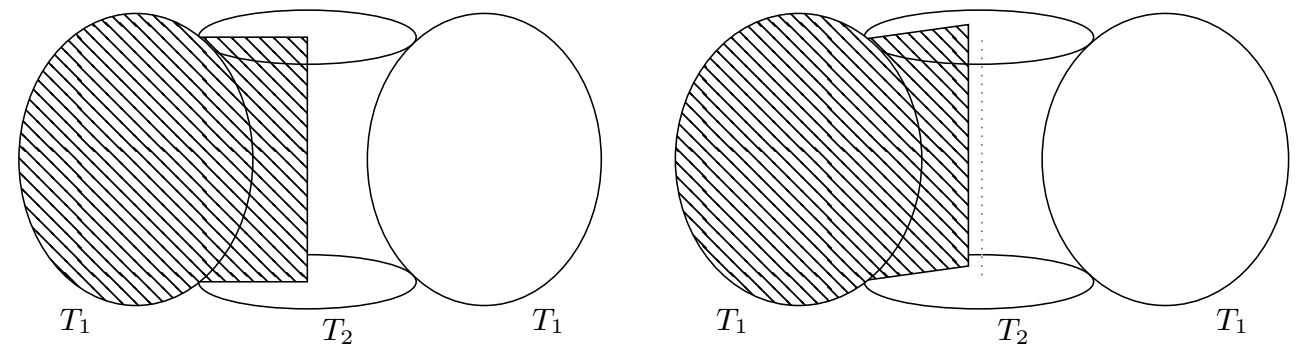

Figure 2: $X_{1,1+j^{\prime}}$ and its perturbation $\widetilde{X}_{1,1+j^{\prime}}$

Proposition 3.29. The boundary of $\widetilde{X}_{1,1+j}$ is $A_{1} \cap \widetilde{A}_{1+j}$.

Proof. The only boundary is $\Gamma\left(\widetilde{\chi}_{1,1+j}^{2}\right)((0, \theta) \times \tau)$, but this is $A_{1} \cap \widetilde{A}_{1+j}$.

Proposition 3.30. When $j$ and $j^{\prime}$ are $q$-covering such that $\left|m-m^{\prime}\right| \in(0, q)_{S^{1}}$, then

$$
A_{1+j^{\prime}} \cap \widetilde{X}_{1,1+j}= \begin{cases}\emptyset, & \text { if } j^{\prime} \text { is not an interloper of } j \\ A_{1+j^{\prime}} \cap S, & \text { if } j^{\prime} \text { is an interloper of } j .\end{cases}
$$

Moreover, in the second case the orientations on $A_{1+j^{\prime}} \cap \widetilde{X}_{1,1+j}$ and $A_{1+j^{\prime}} \cap S$ agree.

We now compute the intersections with $\widehat{X}_{1,1}$ as defined in Definition 3.24. The intersection $A_{\ell} \cap \widehat{X}_{1,1}$, is treated similarly to the previous case.

Proposition 3.31. When $j$ is $q$-covering and $\ell \in \mathbb{Z}_{p}$ then

$$
A_{1+\ell} \cap \widehat{X}_{1,1}= \begin{cases}\emptyset, & \text { if } \ell \text { is not } q \text {-covering } \\ \Gamma\left(\alpha_{\ell}^{2}\right)((0,-, 0) \times \tau), & \text { if } \ell \text { is } q \text {-covering. }\end{cases}
$$

Here $\tau \in I_{\tau}$ where $I_{\tau}$ is as defined in Remark 3.7.

Proposition 3.32. Let $j$ be q-covering. The intersection $A_{1+j} \cap \widehat{X}_{1,1}$, is homologous to $\bigcup_{k} A_{k} \cap S$, where $k$ ranges over all interlopers of $j$. Moreover, the orientations differ by a sign so $\left[A_{1+j} \cap \widehat{X}_{1,1}\right]=-\left[\bigcup_{k} A_{k} \cap S\right]$. 
Proof. Define a bounding manifold $U_{j}(\xi)$, as $\Gamma(\nu)$ for

$$
\begin{aligned}
& \nu=\nu^{1} \cup \nu^{2}:[0,1] \times I \longrightarrow S^{3} \\
& \nu_{j}^{1}:(\xi, \tau) \longmapsto(2 \xi, \tau, \sigma) \quad \text { for } \xi \in\left[0, \frac{1}{2}\right] \\
& \nu_{j}^{2}:(\xi, \tau) \longmapsto(2-2 \xi, \sigma, \tau) \quad \text { for } \xi \in\left[\frac{1}{2}, 1\right]
\end{aligned}
$$

for $\tau \in I_{\tau}, \sigma \in I_{\sigma}$ and $q \sigma=\tau$. The boundary of $U_{j}(\xi)$ is the union $U_{j}(0) \cup U_{j}(1)$, and these components are recognizable as

$$
\begin{aligned}
& U_{j}(0)=A_{1+j} \cap \widehat{X}_{1,1} \\
& U_{j}(1)=\bigcup_{k} A_{k} \cap S .
\end{aligned}
$$

We note that the orientation of the first interval factor induces the difference of orientation between $U_{j}(0)$ and $U_{j}(1)$. Therefore, $A_{1+j} \cap \widehat{X}_{1,1}$ is homologous to $-\left(\bigcup_{k} A_{k} \cap S\right)$.

Combining all of the previous intersection computations we have the main theorem of this section which gives a complete picture of the Massey product structure for $C^{*}\left(\widetilde{\operatorname{Conf}}_{2}(L(p, q))\right)$. In summary we have the following theorem.

Theorem 3.33. After appropriate perturbations, for $j q$-covering and $k$ an interloper of $j$, the intersection $A_{1+k} \pitchfork X_{1,1+j}$ equals $A_{1+k} \pitchfork S$. For $j$ q-covering the intersection $A_{1+j} \pitchfork X_{1,1}$ is homologous to $-\sum_{k} A_{1+k} \pitchfork S$, where the sum ranges over all interlopers of $j$.

We give two examples using this theorem. Theses examples are interesting to compare since the Lens spaces $L(11,2)$ and $L(11,3)$ are the smallest Lens spaces that both admit non-trivial Massey products and are homotopy equivalent but not homeomorphic. The tables below describe only the non-empty intersections $\left(A_{1+\ell} \pitchfork X_{1,1+\ell^{\prime}}\right)$ using the following conventions: if an entry is blank the intersection is empty, and a list of numbers, $i_{1}, \ldots, i_{n}$ represents the intersection $\pm\left(A_{1+i_{1}}+\cdots+A_{1+i_{n}}\right) \cap S$.

\begin{tabular}{|c|c|c|c|}
\hline & $\ell^{\prime}=0$ & $\ell^{\prime}=5$ & $\ell^{\prime}=6$ \\
\hline$\ell=0$ & & 0 & 0 \\
\hline$\ell=1$ & & 1 & \\
\hline$\ell=2$ & & 2 & \\
\hline$\ell=3$ & & 3 & \\
\hline$\ell=4$ & & 4 & \\
\hline$\ell=5$ & $0,1,2,3,4,5$ & 5 & \\
\hline$\ell=6$ & $6,7,8,9,10,0$ & & 6 \\
\hline$\ell=7$ & & & 7 \\
\hline$\ell=8$ & & & 8 \\
\hline$\ell=9$ & & & 9 \\
\hline$\ell=10$ & & & 10 \\
\hline
\end{tabular}

Table 3: Intersections in $L(11,2)$ 


\begin{tabular}{|c|c|c|c|c|c|}
\hline & $\ell^{\prime}=0$ & $\ell^{\prime}=3$ & $\ell^{\prime}=7$ & $\ell^{\prime}=4$ & $\ell^{\prime}=8$ \\
\hline$\ell=0$ & & 0 & 0 & 0 & 0 \\
\hline$\ell=1$ & & 1 & 1 & & \\
\hline$\ell=2$ & & 2 & 2 & & \\
\hline$\ell=3$ & $0,1,2,3$ & 3 & 3 & & \\
\hline$\ell=4$ & $5,6,7,8,9,10,0$ & & 4 & & \\
\hline$\ell=5$ & & & 5 & 5 & \\
\hline$\ell=6$ & & & 6 & 6 & \\
\hline$\ell=7$ & $0,1,2,3,4,5,6,7$ & & 7 & 7 & \\
\hline$\ell=8$ & $8,9,10,0$ & & & 8 & 8 \\
\hline$\ell=9$ & & & & 9 & 9 \\
\hline$\ell=10$ & & & & 10 & 10 \\
\hline
\end{tabular}

Table 4: Intersections in $L(11,3)$

\section{Rational homotopy models}

In the last section we gathered the geometric information necessary to step into the algebraic setting of rational homotopy theory. In this section we build rational models, $A(p, q)$ for the co-chains on $\widetilde{\operatorname{Conf}}_{2}(L(p, q))$.

\subsection{A Rational Co-chain Model}

We now construct a model $A(p, q)$ for the rational co-chains on $\widetilde{\operatorname{Conf}_{2}}(L(p, q))$. Recall the definition of $q$-covering and interloper of $j$ from Definition 3.1.

Definition 4.1. Let $B$ be the free differential graded algebra generated in degree two by $a_{k}$, for $k \in \mathbb{Z}_{p}$, and in degree three by $\eta$ and $x_{j k}$ for $j \leqslant k$ and $j, k \in \mathbb{Z}_{p}$. Define the differential to be $d\left(x_{j k}\right)=a_{j} a_{k}$ and zero otherwise.

Let $A(p, q)$ be the differential graded algebra that is zero in degrees greater than five and in degrees less than or equal to five define $A(p, q)$ to be the quotient of $B$ modulo the following relations:

$\sum_{k \in \mathbb{Z}_{p}} a_{k}=0$

$a_{i} a_{i+\ell}=x_{i, i+\ell}=0$ if $\ell$ is not $q$-covering;

$a_{i+\ell} x_{i, i+j}=0$ for $j q$-covering and $\ell$ not an interloper of $j$;

$a_{i+k} x_{i, i+j}=\eta a_{i+k}$ for $j q$-covering and $k$ an interloper of $j$;

$-a_{i+j} x_{i, i}=\sum_{k} a_{i+k} \eta$, where $j q$-covering and $k$ ranges over all interlopers of $j$.

The following theorem is the main result of this section.

Theorem 4.2. The algebra $A(p, q)$ is a rational model for the cochains of $\widetilde{\operatorname{Conf}}_{2}(L(p, q))$.

This theorem follows from Theorem 5.15 of Halperin and Stasheff $[\mathbf{6}]$ and the Massey product calculations in Section 3. In particular, the only non-zero obstructions of Theorem 5.15 correspond precisely to Massey triple products. 


\subsection{The Homotopy Lie Algebra}

To give generators for the rational homotopy groups we notice that the fibration arising from the projection onto the first coordinate

$$
\bigvee_{p-1} S^{2} \longrightarrow \widetilde{\operatorname{Conf}_{2}}(L(p, q)) \stackrel{p r}{\longrightarrow} S^{3}
$$

has a section. Thus the homotopy long exact sequence splits yielding

$$
\pi_{k}\left(\bigvee_{p-1} S^{2}\right) \hookrightarrow \pi_{k}\left(\widetilde{\operatorname{Conf}}_{2}(L(p, q))\right) \rightarrow \pi_{k}\left(S^{3}\right)
$$

for all $k \geqslant 1$. This implies that the homotopy Lie algebra contains a free Lie algebra on $(p-1)$ generators in degree two. There is also a generator in degree three corresponding to some choice of splitting

$$
\pi_{k}\left(\widetilde{\operatorname{Conf}}_{2}(L(p, q))\right) \hookleftarrow \pi_{k}\left(S^{3}\right) .
$$

Rationally $\pi_{k}\left(S^{3}\right)$ is trivial for $k>3$ which proves the following additive result.

Proposition 4.3. The homotopy Lie algebra of $\pi_{k}\left(\widetilde{\operatorname{Conf}}_{2}(L(p, q))\right)$ is additively given by one generator in degree three and the remaining generators arise from a free Lie algebra with $(p-1)$ generators in degree two.

We note that the additive structure depends only on $p$. The Lie algebra structure however, is significantly more complex. In particular if the generator in degree three is denoted by $S$ and the Lie algebra generators in degree two are denoted by $\alpha_{i}$ then there are relations of the form

$$
\left[\alpha_{i}, S\right]=\sum_{0<j, k, \ell<p} c_{j k \ell}^{i}\left[\left[\alpha_{j}, \alpha_{k}\right], \alpha_{\ell}\right] .
$$

In further work we plan to investigate to what extent the Lie algebra structure can distinguish the homeomorphism type of Lens spaces. In particular we plan to compute the coefficients $c_{j k \ell}^{i}$ and use them to define and compute invariants of the Lie algebra structure.

\section{Acknowledgements}

The author would like to thank Dev Sinha for countless helpful conversations and his continued support throughout this project. We would also like to thank Paolo Salvatore for his comments and for pointing out a sign error in a draft of the paper. We would like to thank the anonymous referee who raised the interesting question stated in introduction and who made suggestions which helped to clarify the location of this work in the literature.

\section{References}

[1] David J. Anick, Hopf algebras up to homotopy, J. Amer. Math. Soc. 2 (1989), no. 3, 417-453. MR 991015 (90c:16007) 
[2] _ R-local homotopy theory, Homotopy theory and related topics (Kinosaki, 1988), Lecture Notes in Math., vol. 1418, Springer, Berlin, 1990, pp. 78-85. MR 1048177 (91c:55008)

[3] Martin Bendersky and Sam Gitler, The cohomology of certain function spaces, Trans. Amer. Math. Soc. 326 (1991), no. 1, 423-440. MR MR1010881 (92d:55005)

[4] F. R. Cohen and L. R. Taylor, Computations of Gel' fand-Fuks cohomology, the cohomology of function spaces, and the cohomology of configuration spaces, Geometric applications of homotopy theory (Proc. Conf., Evanston, Ill., 1977), I, Lecture Notes in Math., vol. 657, Springer, Berlin, 1978, pp. 106-143. MR MR513543 (80f:58050)

[5] Yves Felix and Jean-Claude Thomas, Configuration spaces and massey products, Int. Math. Res. Not. (2004), no. 33, 1685-1702. MR MR2058024 (2005b:55039)

[6] Stephen Halperin and James Stasheff, Obstructions to homotopy equivalences, Adv. in Math. 32 (1979), no. 3, 233-279. MR MR539532 (80j:55016)

[7] Pascal Lambrechts and Don Stanley, The rational homotopy type of configuration spaces of two points, Ann. Inst. Fourier (Grenoble) 54 (2004), no. 4, 1029-1052. MR MR2111020 (2005i:55016)

[8] Riccardo Longoni and Paolo Salvatore, Configuration spaces are not homotopy invariant, Topology 44 (2005), no. 2, 375-380. MR MR2114713 (2005k:55024)

[9] Matthew S. Miller, The rational homotopy type of configuration spaces of threedimensional lens spaces, Ph.D. thesis, University of Oregon, 2007.

[10] Kurt Reidemeister, Complexes and homotopy chains, Bull. Amer. Math. Soc. 56 (1950), 297-307. MR MR0036506 (12,120b)

[11] Burt Totaro, Configuration spaces of algebraic varieties, Topology 35 (1996), no. 4, 1057-1067. MR MR1404924 (97g:57033)

Matthew S. Miller mamiller@vassar.edu

Mathematics Department, Vassar College, Poughkeepsie, NY, 12604, U.S.A 